\title{
ARTÍCULOS ORIGINALES \\ Mejoramiento continuo de la atención prenatal y su impacto en la mortalidad perinatal en dos instituciones de salud de Popayán
}

Ivan Jaramillo Arias*, Alba Inés Palomino**, Sonia Pasmiño de Osorio ${ }^{* * *}$, Fernando Pinzón B. *****

\section{RESUMEN}

OBJETIVO: Evaluar el impacto de un modelo de Mejoramiento Continuo de la Atención Prenatal sobre la morbilidad materna y la morbimortalidad Perinatal.

DISEÑO: Estudio prospectivo descriptivo.

MATERIALES Y METODOS: Entre Enero de 1997 y Mayo de 1998, se evaluó la calidad de la atención ofrecida a las pacientes que asistieron a control prenatal en el Centro de Atención Ambulatoria del Instituto de Seguros Sociales (CAA - ISS) y el Centro de Salud Suroccidente del Servicio de Salud del Cauca (CSS -SS) de la ciudad de Popayán. Después de un período de observación de cinco meses, la intervención, realizada en los doce meses siguientes, consistió en la aplicación de un modelo de control de calidad, utilizando un formato diseñado para evaluar el correcto diligenciamiento de 92 variables de la Historia Clínica Perinatal, que permitía además la supervisión y la asesoría continua del personal que realizaba el control. Se analizó el diligenciamiento de 22.292 variables que correspondieron a una muestra del $5.7 \%$ de los controles prenatales realizados en ambas Instituciones. El impacto de la intervención se evaluó en los resultados de 1.217 eventos obstétricos ocurridos durante el período del estudio.

RESULTADOS: El diligenciamiento correcto de todas las variables se logró incrementar de un 65\% a un 92.2\% en el CAA ISS y del $84.5 \%$ al $90.8 \%$ en el CSS - SS. El mayor impacto de la intervención se evidenció en las tasas de mortalidad perinatal que disminuyeron de 24.3 a 13.0 en el CAA - ISS y de 12.7 a 0 en el CSS - SS, con coeficientes de correlación lineal estadísticamente significativos especialmente en el Seguro Social.

CONCLUSIONES: El buen diligenciamiento de la historia clínica, la aplicación de un instrumento de control de calidad y la supervisión conducen al mejoramiento de los resultados perinatales.

PALABRAS CLAVES: Control Prenatal, Control de Calidad, Mortalidad Perinatal

Médico Ginecobstetra. Universidad del Cauca.

Médica Ginecobstetra. Universidad del Cauca.

$\begin{array}{ll}* * * & \text { Medica Ginecobstetra. Universidad del Valle. Coordinadora del Programa. } \\ * * * * & \text { Biólogo _ Salubrista. Facultad de Ciencias de la Salud. Universidad del Cauca. }\end{array}$ 


\section{SUMARY}

OBJECTIVE: Evaluate the impact of a Prenatal care continuous improvement model on both maternal morbidity and perinatal morbidity and mortality.

DESIGN: Prospective descriptive study.

MATHERIALS AND METHODS: Care quality offered to patients attending perinatal control at CAA - ISS (Centro de atención ambulatoria Popayán - Instituto de Seguros Sociales) and CSS - SS (Centro de Salud Suroccidente Popayán - Servicio de Salud del Cauca) between January 1997 and May 1998 was evaluated. Following a 5 month observation period, the 12 month intervention consisted on the application of a quality control model using a format deisgned to evaluate the proper filling out of 92 variables on the Perinatal clinical record, which also allowed the supervision and continuos assistance of the personnel performing the controls. Filling out of 22.292 variables corresponding to a 5.7\% prenatal control sample fron both institutions was evaluated. The intervention impact was evaluated on the outccmes from 1217 obstetric events ocurred during the period of study.

RESULTS: Proper filling out of every variable increased from $65 \%$ to $92.2 \%$ at CAA - ISS and $84.5 \%$ to $90.8 \%$ at CSS - SS.The intervention most striking impact was shown on perinatal mortality rates which decreased from 24.3 to 13.0 at CAA - ISS and from 12.7 to 0 at CSS - SS, with statistically significant linear correlation coefficients particulary at ISS.

CONCLUSIONS: Proper filling out of the medical record, applying a quality control instrument and personnel supervising all lead to perinatal outcome improvement.

KEY WORDS: Prenatal Care, Quality control, Perinatal Mortality.

$$
\begin{array}{r}
\text { " Y así para los dos, el hijo muerto, } \\
\text { que fue en nosotros la ilusión más bella, } \\
\text { tenía el hechizo rubio de una estrella, } \\
\text { y la fragancia del pomar del huerto... } \\
\text { Ya no llores amada, une tu pena, } \\
\text { a mi trágica herida nazarena, } \\
\text { y mientras yo en la evocación me pierdo, } \\
\text { y en ti se aviva del dolor la ola, } \\
\text { mezamos otra vez la cuna sola, } \\
\text { y verás que se duerme su recuerdo." } \\
\text { BAUDILIO MONTOYA } \\
\text { POETA QUINDIANO }
\end{array}
$$

\section{BAUDILIO MONTOYA POETA QUINDIANO}

\section{INTRODUCCIÓN}

La muerte materna y la muerte fetal se erigen como dos tragedias propias del subdesarrollo en las que alguna vez como médicos hemos sido testigos o protagonistas. Constituyen dos situaciones extremadamente tristes y dolorosas, de graves repercusiones sociales y psicológicas para el grupo familiar que sobrevive y que trascienden como el resultado final de una serie de circunstancias negativas que van desde la discriminación, la pobreza, la ignorancia, y la falta de recursos hasta la carencia tecnológica y el desacierto o la negligencia en el actuar médico.
Las tasas de mortalidad materna y perinatal se aceptan como indicadores que reflejan la eficiencia en la atención que se le brinda a la mujer embarazada ${ }^{1-2}$, están en estrecha relación con determinantes sociales, económicos y demográficos y son el reflejo de la calidad de vida de cada país ${ }^{3}$. La desigualdad entre las naciones, la discriminación y las grandes diferencias sociales aún dentro de un mismo país y región son determinantes que establecen grandes variaciones en la mortalidad materna y perinatal, incluso entre ciudades e instituciones ${ }^{2,4-7}$.

A pesar de su enorme validez, la mayoría de los países de América Latina carecen de tasas de mortalidad perinatal confiables, existiendo grandes subregistros y la tendencia a reportar con mayor frecuencia tasas hospitalarias que tienen un amplio rango de variación entre 18 y 40 por mil nacimientos $^{8}$, alcanzando en algunas Instituciones cifras extremadamente elevadas de hasta 49 por mil 2.

La salud perinatal guarda una relación íntima con múltiples factores sociales, culturales, genéticos, económicos y ambientales, pero quizá sea la salud integral de la madre aún desde la etapa preconcepcional y la utilización oportuna y adecuada de servicios médicos prenatales y neonatales de alta calidad, los factores más susceptibles de modificar y 
de mayor incidencia en las tasas de mortalidad perinatal. Por tal razón los indicadores específicos para evaluar los programas orientados a su reducción apuntan hacia el mejoramiento de los programas de planificación familiar, nutrición, control prenatal y parto 8 .

Aunque la cobertura del control prenatal en Colombia ha aumentado considerablemente en las últimas décadas ${ }^{9}$, es frecuente sin embargo, aún hoy en día, la captación tardía en el último trimestre del embarazo, un bajo promedio de controles prenatales por paciente y el llenado incompleto de la Historia clínica ${ }^{10}$ que se ha convertido en una desafortunada costumbre en la práctica médica moderna.

Se ha sugerido que un primer paso en el mejoramiento de la atención a la mujer embarazada es un adecuado y cuidadoso control prenatal ${ }^{10}$ y el mejoramiento en la calidad de la historia clínica ha demostrado ser eficaz en disminuir la mortalidad materna y la morbimortalidad perinatal en dos investigaciones previas ${ }^{11-12}$ que utilizaron la misma metodología de este estudio.

La presente investigación fue diseñada como parte de un Estudio Colaborativo Nacional auspiciado por la Sociedad Colombiana de Obstetricia y Ginecología con el objetivo de verificar el impacto de la intervención en la calidad del control prenatal y el correcto diligenciamiento de la historia sobre la morbilidad materna y la morbilidad y mortalidad perinatal.

\section{MATERIALES Y MÉTODOS}

Entre Enero de 1997 y Mayo de 1998 se realizó un estudio prospectivo de las pacientes gestantes que asistieron a control prenatal en el Centro de Atención Ambulatoria del Seguro Social (CAA - ISS) y en el Centro de Salud Suroccidente-Unidad Popayán del Servicio de Salud del Cauca (CSS - SS) en la ciudad de Popayán.

Durante un período inicial de cinco meses se realizó una observación de la calidad de la atención y el desempeño de los profesionales encargados del control prenatal, evaluando el estado de los instru-mentos de medición como balanzas y tensiómetros, así como el comportamiento del recurso humano con relación al cumplimiento de las normas y al completo y correcto diligenciamiento de la historia clínica. Durante este mismo período se evaluó el resultado de los eventos obstétricos de cada institución, así como los índices de patología materna y del recién nacido.

Durante los 12 meses siguientes un ginecobstetra visitó cada una de las instituciones del estudio al menos una vez por semana, en días distintos y en horarios no programados, seleccionando al azar de cinco a diez historias clínicas de las pacientes en consulta, revisando y registrando los defectos en un instrumento diseñado con 92 variables, calificando la calidad del registro de acuerdo a una puntuación preestablecida y siguiendo el siguiente esquema:

- 0 = Cero puntos. La variable fue registrada completamente, se reconoció el riesgo si lo había y se promovieron las acciones correspondientes.

- 1 = Un punto. Se registró la casilla, se reconoció el riesgo, pero no se originaron acciones

- 2 = Dos puntos. La casilla se registró correctamente, pero no se registró el riesgo ni se originaron acciones.

- 3 = Tres puntos. Casilla totalmente en blanco. No hay dato ni se reconoció el riesgo. No se originaron acciones.

- 4 = Cuatro puntos. Error en el registro o en la interpretación del dato anotado en la casilla.

Las variables fueron agrupadas en identificación, antecedentes, examen \& diagnóstico, exámenes de laboratorio y datos propios del control prenatal durante un máximo de ocho controles. En identificación se incluyó la raza, edad, alfabetización, estudios, años aprobados, estado civil, ocupación y jornada laboral. En antecedentes se agruparon los antecedentes familiares, personales y obstétricos, historia de un recién nacido de bajo peso, fecha del embarazo anterior, periodo intergenésico, peso previo y habito de fumar. En examen \& diagnóstico se catalogaron las variables talla, fecha de última regla, fecha probable del parto, examen clínico, odontológico, de cervix y de mamas. En exámenes de laboratorio se incluyeron hemoclasificación, serología, vacunación antitetánica, citología, glicemia, hemoglobina \& hematocrito, uroanálisis y otros exámenes. Finalmente en cada control prenatal se incluyeron como variables, edad gestacional, peso, tensión arterial, altura uterina, concordancia amenorrea y altura uterina, fetocardia y presentación, además de las gráficas de incremento de peso y altura uterina en función de la edad gestacional.

La calificación del sistema fue diseñada para castigar con una puntuación más alta la omisión, el error o la falta de actuar médico frente a las situaciones de riesgo, mientras que una puntuación baja era el reflejo de menos errores, buena calidad y diligencia en la atención.

En los casos de manejo inadecuado se brindó asesoría directa al profesional tratante y se realiza- 
ron reuniones con los médicos y directivos de las instituciones para estandarizar procedimientos y analizar los resultados encontrados. De igual forma se revisó periódicamente el funcionamiento de los instrumentos de medición empleados en la consulta prenatal.

Puesto que ninguna de las dos instituciones incluidas en el estudio presta atención directa para el parto, fue necesario revisar todos los partos atendidos en todas las Instituciones de Salud de la ciudad de Popayán para determinar la finalización y los resultados de cada uno de los eventos obstétricos.

En el estudio se incluyeron todas las embaraza-das que asistieron a control prenatal al CAA - ISS y al CSS SS. En el CAA _ ISS se concentra la mayor parte del control prenatal de las embarazadas afiliadas y beneficiarias del Seguro Social de Popayán. El CSS - SS presta atención a una gran población de recursos medios y bajos del sector suroccidental de la ciudad y tiene además una importante afluencia de pacientes de los municipios vecinos del sur del Departamento. En ambas instituciones el control prenatal lo realiza básicamente el médico general y una enfermera y los embarazos complicados o de alto riesgo se remiten al médico especialista.

La evaluación de los resultados se hizo a través de las variaciones en el buen diligenciamiento de la Historia clínica, de la cuantificación de los defectos en el llenado de la misma y de los errores en la identificación del riesgo $o$ en la conducta médica asumida frente al riesgo obstétrico detectado.

El impacto de la intervención se analizó a través de las variaciones en la patología asociada al embarazo, en la morbilidad del recién nacido, en la mortalidad perinatal, en el número de traslados de los recién nacidos a la Unidad de Neonatología y en la patología presente en el momento del nacimiento. Los eventos obstétricos fueron analizados en el Sistema Informativo Perinatal del Centro Latinoamericano de Perinatología CLAP _ OPS / OMS y las variables fueron sometidas a un análisis estadístico para establecer su significancia utilizando el paquete estadístico MICROSTAT para el análisis bivariado de los resultados.

\section{RESULTADOS}

Durante el período de observación de cinco meses comprendido entre el $1^{\circ}$ de enero y el 30 de mayo de 1997 se analizaron 415 eventos obstétricos que correspondieron aproximadamente al $44.8 \%$ de las pacientes controladas en las dos Instituciones del estudio en ese mismo período. Durante la fase de intervención de doce meses comprendidos entre el $1^{\circ}$ de junio de 1997 y el 30 de mayo de 1998, se evaluó la calidad de la atención en 1.865 pacientes que realizaron7.316 controles prenatales con un promedio de 3.9 consultas por paciente. Se monitorizaron durante el control prenatal 417 historias clínicas que correspondieron al $5.7 \%$ de los controles realizados, analizando el diligenciamiento de 22.292 variables. Durante este mismo período se evaluó el resultado de 802 eventos obstétricos que correspondieron al $52.7 \%$ del total de partos esperados para ese período.

En la fase inicial del estudio se constató que los consultorios médicos carecían en ambas instituciones de muchos de los elementos necesarios para un adecuado control prenatal y algunos de ellos se encontraban en mal estado o estaban dañados. En ninguna de las instituciones se calibraban las balanzas y los tensiómetros.

Los consultorios del CSS - SS fueron equipados y adecuados de acuerdo a los requisitos mínimos solicitados y se dispuso durante el seguimiento de instrumentos de medición calibrados y en buen estado. En el CAA - ISS fue mucho más difícil cumplir este objetivo por una supuesta carencia de recursos económicos a pesar de que los implementos solicitados requerían una mínima inversión.

Los principales defectos observados duran-te el seguimiento fueron la variable no diligenciada y el error en el registro. Solo el $65 \%$ de las variables eran correctamente diligenciadas en el CAA - ISS en el primer trimestre del estudio, lográndose durante la intervención el registro correcto del $92.2 \%$ de todas las variables en último trimestre. (Tabla 1). El mal diligenciamiento de la historia, inicialmente observado en el CAA - ISS es el reflejo de lo que ocurría en esa institución donde se llevaban al menos tres modelos distintos de historia prenatal y existía la desatinada tendencia del personal médico a no escribir los datos del control prenatal en la historia diseñada para tal efecto, sino en las notas de evolución o en hojas distintas, situación que conducía a frecuentes omisiones en los datos y errores en las apreciaciones. Al adoptar el Seguro Social en los primeros meses del estudio una historia clínica única, se observa una mejoría progresiva en la eficiencia que va siendo más notable a medida que el personal médico aprende y se familiariza conel nuevo formato.

Se observó un mejor diligenciamiento de la Historia clínica en el CSS -SS en el primer trimestre del estudio, lo cual era de esperarse pues un modelo único de historia prenatal había sido implantado en esa institución desde hacía varios años. Sin embargo la cifra inicial reflejaba que las normas establecidas 
Tabla 1.

Porcentaje de variables bien diligenciadas. CAA - ISS. Período de intervención junio de 1997 - mayo de 1998

\begin{tabular}{|lcccc|}
\hline VARIABLE & $\begin{array}{c}\text { PRIMER } \\
\text { TRIMESTRE } \\
\mathrm{n}=2.926\end{array}$ & $\begin{array}{c}\text { SEGUNDO } \\
\text { TRIMESTRE } \\
\mathrm{n}=3.555\end{array}$ & $\begin{array}{c}\text { TERCER } \\
\text { TRIMESTRE } \\
\mathrm{n}=3.576\end{array}$ & $\begin{array}{c}\text { CUARTO } \\
\text { TRIMESTRE } \\
\mathrm{n}=3.692\end{array}$ \\
\hline IDENTIFICACIÓN & 49.3 & 68.0 & 78.0 & 89.6 \\
ANTECEDENTES & 61.4 & 74.6 & 85.9 & 93.5 \\
EXAMEN \& DIAGNÓSTICO & 53.0 & 73.3 & 76.7 & 79.4 \\
LABORATORIOS & 76.9 & 74.3 & 79.9 & 85.8 \\
PRIMER CONTROL & 64.7 & 82.9 & 90.9 & 97.9 \\
TODAS LAS VARIABLES & 65.8 & 79.1 & 85.8 & 92.2 \\
\hline
\end{tabular}

Tabla 2.

Porcentaje de variables bien diligenciadas. CSS - SS. Período de intervención junio de 1997 _ mayo de 1998

\begin{tabular}{|lcccc|}
\hline VARIABLE & $\begin{array}{c}\text { PRIMER } \\
\text { TRIMESTRE } \\
\mathrm{n}=3.161\end{array}$ & $\begin{array}{c}\text { SEGUNDO } \\
\text { TRIMESTRE } \\
\mathrm{n}=1.891\end{array}$ & $\begin{array}{c}\text { TERCER } \\
\text { TRIMESTRE } \\
\mathrm{n}=1.795\end{array}$ & $\begin{array}{c}\text { CUARTO } \\
\text { TRIMESTRE } \\
\mathrm{n}=1.696\end{array}$ \\
\hline IDENTIFICACIÓN & 86.0 & 90.3 & 90.6 & 91.6 \\
ANTECEDENTES & 85.8 & 90.1 & 95.6 & 94.5 \\
EXAMEN \& DIAGNÓSTICO & 81.3 & 90.8 & 82.8 & 83.5 \\
LABORATORIOS & 88.4 & 93.7 & 87.6 & 83.8 \\
PRIMER CONTROL & 75.8 & 93.3 & 87.4 & 93.9 \\
TODAS LAS VARIABLES & 84.5 & 93.0 & 91.3 & 90.8 \\
\hline
\end{tabular}

con relación al llenado de la historia clínica no se cumplían en su totalidad. Durante la intervención en esta institución se logró mejorar el índice de variables bien diligenciadas desde un valor inicial de $84.5 \%$ a una cifra de $90.8 \%$ para todas las variables al término del estudio. (Tabla 2).

A pesar de que la tendencia al mejoramiento fue similar en ambas instituciones el CAA - ISS mostró una mejoría más uniforme y sostenida. Las fluctua- ciones en la calidad del llenado de la historia en el CSS - SS fueron atribuidas a cambios del personal médico y modificaciones en las políticas de la Institución con relación al subsidio de los exámenes de laboratorio de la embarazada.

Los principales errores correspondieron a la falta de llenado o llenado incorrecto de las gráficas de altura uterina e incremento de peso en función de la edad gestacional, pues ninguna de las instituciones 
diligenciaba esta variable al comienzo del estudio. En el primer trimestre de la intervención se observó igualmente que existían frecuentes errores en la estimación de la edad gestacional, cifra que se reduce notablemente en ambas instituciones al término de la intervención (Figura 004i01).

La media de la edad materna fue de 23.8 en el CSS - SS (DS: 6.2) y 26.4 en el CAA - IS (DS: 6.3). Al evaluar la patología materna se observa que más

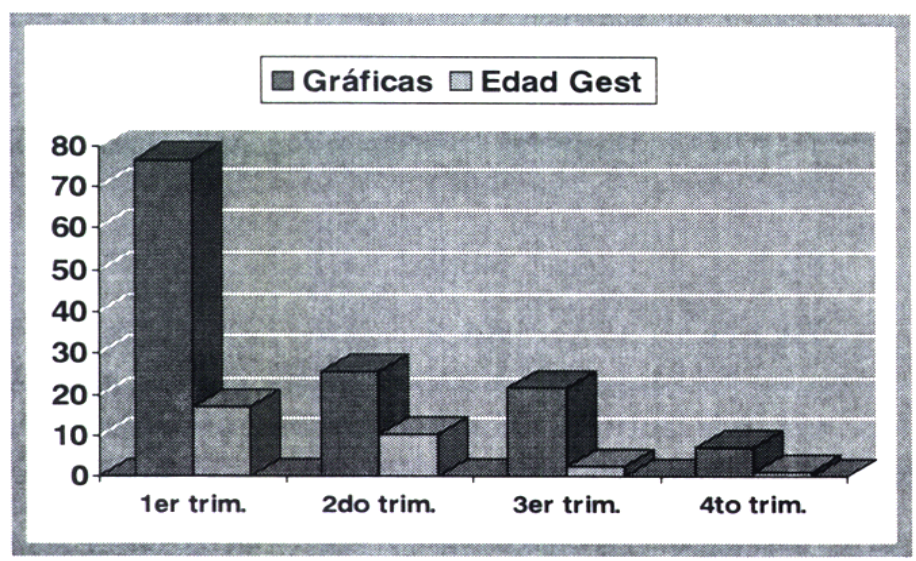

Figura $004 \mathrm{i} 01$

Principales variables mal diligenciadas: Falta de llenado o error en el registro de gráficas de peso - altura uterina y cálculo de la edad gestacional, expresado en porcentajes. de $50 \%$ de las mujeres embarazadas presentan algún tipo de morbilidad durante su gestación, siendo notable un incremento de la patología materna en el CAA-ISS posiblemente como consecuencia de una mejor detección. Ambas Instituciones muestran a través del estudio una frecuencia mayor en el diagnóstico de amenaza de parto pretérmino y desproporción fetopélvica, así como una tendencia a la disminución en la incidencia de preeclampsia. (Tablas 3 y 4). No se registraron muertes maternas.

El elevado índice de cesáreas, que fluctua entre $28 \%$ y $62 \%$ a lo largo del estudio no muestra una tendencia racional con los niveles de complejidad de las instituciones que prestan la atención obstétrica. Aunque la decisión del procedimiento debe corresponder a un criterio científico real y como lo recomienda la Organización Mundial de la Salud (OMS) su número no debe sobrepasar el límite de $15 \%$ de los partos ${ }^{9}$, ha calado en nuestro medio una estrategia facilista que impone la cesárea como la mejor conducta para solucionar cualquier complicación eventual del embarazo.

El 54\% de los recién nacidos fue de sexo masculino en el CSS-SS y el $49 \%$ en el CAA-ISS y el peso promedio fue de 3.140 gramos en el CSS-SS (DS.470 gr.) y 3.145 gramos en el CAA-ISS (DS.540 gr.) La patología del recién nacido mostró una tendencia a disminuir a lo largo del estudio y en ambas instituciones se redujo el número de neonatos deprimidos,

Tabla 3.

Patología materna CAA - ISS. enero de 1997 _ mayo de 1998

\begin{tabular}{|c|c|c|c|c|c|}
\hline $\begin{array}{l}\text { VARIABLE } \\
\\
\mathrm{PI}= \\
\mathrm{PI}\end{array}$ & $\begin{array}{l}\text { ERIODO } \\
\text { REVIO } \\
=336 \\
\%\end{array}$ & $\begin{array}{c}\text { PRIMER } \\
\text { TRIMESTRE } \\
\mathrm{n}=151 \\
\%\end{array}$ & $\begin{array}{c}\text { SEGUNDO } \\
\text { TRIMESTRE } \\
\mathrm{n}=135 \\
\%\end{array}$ & $\begin{array}{c}\text { TERCER } \\
\text { TRIMESTRE } \\
\mathrm{n}=135 \\
\%\end{array}$ & $\begin{array}{c}\text { CUARTO } \\
\text { TRIMESTRE } \\
\mathrm{n}=154 \\
\%\end{array}$ \\
\hline ALGUNA PATOLOGÍA & 58.3 & 55.0 & 60.0 & 56.3 & 72.7 \\
\hline INFECCIÓN URINARIA & 11.0 & 11.3 & 8.9 & 5.2 & 16.9 \\
\hline PREECLAMPSIA & 10.1 & 5.3 & 5.2 & 6.7 & 3.9 \\
\hline AMENAZA PARTO PRET. & 5.7 & 4.6 & 2.2 & 3.7 & 9.7 \\
\hline DESPROPORCIÓN & 9.5 & 3.3 & 5.9 & 11.9 & 11.7 \\
\hline R.P.M. & 3.6 & 4.0 & 1.5 & 2.2 & 1.9 \\
\hline CESÁREA & 54.2 & 28.5 & 29.6 & 40.7 & 62.3 \\
\hline
\end{tabular}


Tabla 4.

Patología materna CSS - SS., enero de 1997 _ mayo de 1998

\begin{tabular}{|c|c|c|c|c|c|}
\hline VARIABLE & $\begin{array}{c}\text { PERIODO } \\
\text { PREVIO } \\
\mathrm{n}=79 \\
\%\end{array}$ & $\begin{array}{c}\text { PRIMER } \\
\text { TRIMESTRE } \\
\mathrm{n}=64 \\
\%\end{array}$ & $\begin{array}{c}\text { SEGUNDO } \\
\text { TRIMESTRE } \\
\mathrm{n}=59 \\
\%\end{array}$ & $\begin{array}{c}\text { TERCER } \\
\text { TRIMESTRE } \\
\mathrm{n}=49 \\
\%\end{array}$ & $\begin{array}{c}\text { CUARTO } \\
\text { TRIMESTRE } \\
\mathrm{n}=55 \\
\%\end{array}$ \\
\hline ALGUNA PATOLOGÍA & 53.2 & 43.8 & 45.8 & 49.0 & 52.7 \\
\hline INFECCIÓN URINARIA & 16.5 & 7.8 & 16.9 & 4.1 & 7.3 \\
\hline PREECLAMPSIA & 10.1 & 4.7 & 1.7 & 8.2 & 5.5 \\
\hline AMENAZA PARTO PRET & 2.5 & 1.6 & 3.4 & 8.2 & 12.7 \\
\hline DESPROPORCIÓN & 7.6 & 4.7 & 5.1 & 6.1 & 7.3 \\
\hline R.P.M. & 1.3 & 6.3 & 3.4 & 0 & 1.8 \\
\hline CESÁREA & 29.1 & 43.8 & 22.0 & 28.6 & 40.0 \\
\hline
\end{tabular}

siendo esta tendencia mas notable en el CAA-ISS, donde fue evidente también una marcada reducción en el número de recién nacidos trasladados a la Unidad de Neonatología (Tablas 5 y 6).

El mayor impacto de la intervención se evidenció sobre las tasas de mortalidad perinatal definida como toda muerte del producto de la concepción ocurrida a partir de la semana 28 de gestación hasta el séptimo día del nacimiento. Se encontró una significativa disminución en el número de muertes perinatales cuya cifra logró reducirse desde una tasa inicial de 12.7 por mil nacimientos, a cero en el CSS - SS y de 24.3 a 13.0 en el CAA - ISS. Las variaciones en las tasas de mortalidad perinatal en todo el grupo se ilustran en la Figura 004i02.

Mediante el análisis bivariado de los datos se calcularon los coeficientes de correlación lineal entre las seis variables independientes (variables bien diligenciadas) y todas las quince variables dependientes (patología materna, patología del recién nacido, traslados y mortalidad perinatal) en cada una de las instituciones. Se encontraron valores del coeficiente de correlación lineal estadísticamente significativos a un nivel de $5 \%$ para las variables que se relacionan en las Tablas 7 y 8 , destacándose que el mejor diligenciamiento de las variables del primer control, exámen \& diagnostico y de todas las variables muestra igualmente una correlación estadísticamente significativa con la disminución de la mortalidad perinatal en el CAA-ISS. El mejor diligenciamiento del primer

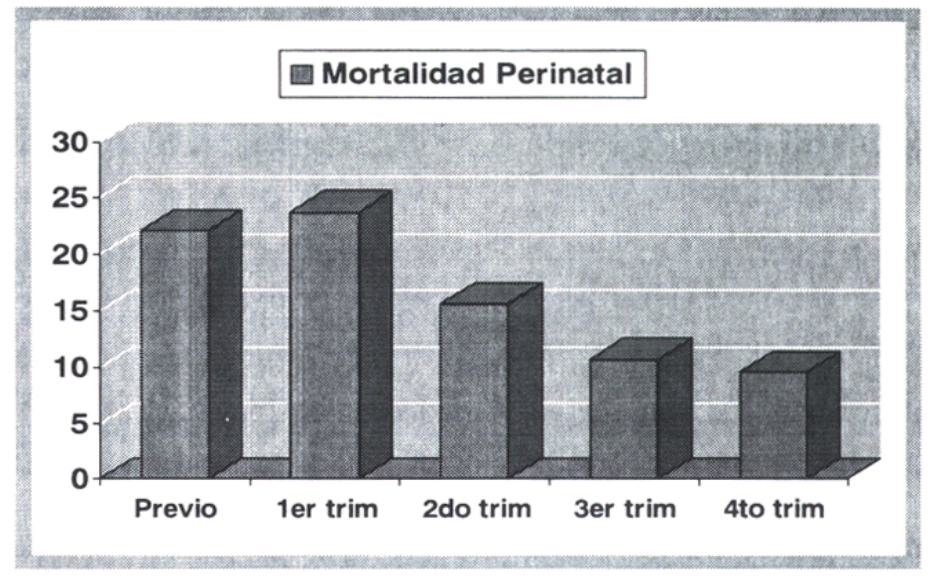

Figura 004i02

Tasas de Mortalidad Perinatal por 1000 nacidos vivos en las dos Instituciones del estudio. Enero de 1997 a mayo de 1998.

control y de todas las variables muestra igualmente una correlación significativa con la disminución de la patología del recién nacido en el CSS -SS.

\section{DISCUSIÓN}

El Ministerio de Salud mediante decreto $\mathrm{N}^{\circ}$ 2174 de noviembre de 1996, expidió una serie de normas y sanciones orientadas a garantizar parámetros mínimos de calidad en la atención en 
Tabla 5.

Patología del recién nacido CAA - ISS., enero de 1997 _ mayo de 1998

\begin{tabular}{|c|c|c|c|c|c|}
\hline VARIABLE & $\begin{array}{c}\text { PERIODO } \\
\text { PREVIO } \\
\mathrm{n}=336 \\
\%\end{array}$ & $\begin{array}{c}\text { PRIMER } \\
\text { TRIMESTRE } \\
\mathrm{n}=151 \\
\%\end{array}$ & $\begin{array}{c}\text { SEGUNDO } \\
\text { TRIMESTRE } \\
\mathrm{n}=135 \\
\%\end{array}$ & $\begin{array}{c}\text { TERCER } \\
\text { TRIMESTRE } \\
\mathrm{n}=135 \\
\%\end{array}$ & $\begin{array}{c}\text { CUARTO } \\
\text { TRIMESTRE } \\
\mathrm{n}=154 \\
\%\end{array}$ \\
\hline ALGUNA PATOLOGÍA & 14.6 & 8.8 & 8.3 & 7.5 & 11.2 \\
\hline HIPERBILIRRUBINEMIA & 1.8 & 0 & 2.3 & 1.5 & 1.3 \\
\hline MEMBRANA HIALINA & 0.6 & 0.7 & 0 & 0 & 0 \\
\hline INFECCIÓN & 0.3 & 0 & 0 & 0.8 & 0.7 \\
\hline DEPRIMIDOS & 6.9 & 2.6 & 2.2 & 3.0 & 5.2 \\
\hline DEFECTOS CONGÉNITOS & 2.4 & 0 & 0 & 0 & 3.3 \\
\hline TRASLADOS & 10.1 & 9.2 & 7.4 & 0.7 & 2.6 \\
\hline MORTALIDAD PERINATAL* & 24.3 & 27.2 & 15.0 & 15.0 & 13.0 \\
\hline
\end{tabular}

* Tasa de mortalidad por 1000 nacidos vivos.

Tabla 6.

Patología del recién nacido CSS - SS., enero de 1997 _ mayo de 1998

\begin{tabular}{|lccccc|}
\hline VARIABLE & $\begin{array}{c}\text { PERIODO } \\
\text { PREVIO } \\
\mathrm{n}=79 \\
\%\end{array}$ & $\begin{array}{c}\text { PRIMER } \\
\text { TRIMESTRE } \\
\mathrm{n}=64\end{array}$ & $\begin{array}{c}\text { SEGUNDO } \\
\text { TRIMESTRE } \\
\mathrm{n}=59\end{array}$ & $\begin{array}{c}\text { TERCER } \\
\text { TRIMESTRE } \\
\mathrm{n}=49\end{array}$ & $\begin{array}{c}\text { CUARTO } \\
\text { TRIMESTRE } \\
\mathrm{n}=55\end{array}$ \\
\hline ALGUNA PATOLOGÍA & 10.3 & 15.9 & 6.9 & 10.2 & 9.1 \\
HIPERBILIRRUBINEMIA & 2.6 & 4.8 & 1.7 & 2.0 & 3.6 \\
MEMBRANA HIALINA & 0 & 1.6 & 0 & 2.0 & 0 \\
INFECCIÓN & 0 & 1.6 & 1.7 & 2.0 & 0 \\
DEPRIMIDOS & 6.4 & 7.8 & 5.1 & 2.0 & 1.8 \\
DEFECTOS CONGÉNITOS & 5.1 & 0 & 1.7 & 0 & 0 \\
TRASLADOS & 5.0 & 14.0 & 5.0 & 6.1 & 14.5 \\
MORTALIDAD PERINATAL* & & & & & \\
\hline
\end{tabular}

\footnotetext{
* Tasa de mortalidad por 1000 nacidos vivos.
} 
Tabla 7.

Correlación de variables estadísticamente significativas CAA_ ISS.

\begin{tabular}{|llr|}
\hline VARIABLE INDEPENDIENTE & VARIABLE DEPENDIENTE & $\mathrm{R}$ \\
\hline IDENTIFICACION & Vs. DESPROPORCION FETOPELVICA & 0.93 \\
ANTECEDENTES & Vs. DESPROPORCION FETOPELVICA & 0.96 \\
EXAMEN \& DIAGNOSTICO & Vs. MEMBRANA HIALINA & -0.98 \\
EXAMEN \& DIAGNOSTICO & Vs. MORTALIDAD PERINATAL & -0.99 \\
PRIMER CONTROL & Vs. DESPROPORCION FETOPELVICA & 0.93 \\
PRIMER CONTROL & Vs. MORTALIDAD PERINATAL & -0.95 \\
TODAS LAS VARIABLES & Vs. DESPOROPORCION FETOPELVICA & 0.93 \\
TODAS LAS VARIABLES & Vs. MORTALIDAD PERINATAL & -0.93 \\
\hline
\end{tabular}

Tabla 8.

Correlación de variables estadísticamente significativas CSS - SS.

\begin{tabular}{|llr|}
\hline VARIABLE INDEPENDIENTE & VARIABLE DEPENDIENTE & $\mathrm{R}$ \\
\hline ANTECEDENTES & Vs. RUP. PREMATURA DE MEMBRANAS & -0.98 \\
ANTECEDENTES & Vs. DEPRIMIDOS & -0.99 \\
EXAMEN \& DIAGNOSTICO & Vs. DEFECTOS CONGENITOS & 0.98 \\
PRIMER CONTROL & Vs. ALGUNA PATOLOGIA (R.N.) & -0.96 \\
TODAS LAS VARIABLES & Vs. ALGUNA PATOLOGIA (R.N.) & -0.98 \\
\hline
\end{tabular}

salud como un primer paso para iniciar un proceso de mejoramiento continuo de los servicios de salud y promover el desarrollo de una cultura de calidad entre los usuarios y las entidades que constituyen el sistema general de seguridad social en salud 13 .

Las Entidades Promotoras de Salud y las Instituciones prestadoras de Servicios, de acuerdo a lo que pretende el Estado en este contexto, deben desarrollar sistemas de garantía de calidad y adelantar acciones de autoevaluación para garantizar eficacia, eficiencia, seguridad y racionalidad técnica en sus servicios.

Un requisito mínimo de calidad es una historia prenatal única, completa, de fácil elaboración, que permita cuantificar el riesgo y que brinde la posibilidad de conocer las estadísticas de cada institución para modificar conductas y corregir errores. No se justifica actualmente continuar probando nuevos y extensos modelos de Historia Prenatal cuando existen instrumentos suficientemente utilizados como el propuesto por el Centro Latinoamericano de Perinatología y
Desarrollo Humano (CLAP) que ha demostrado excelentes resultados en el diagnóstico prenatal y en la identificación de los factores de riesgo 14.

La historia clínica bien diligenciada permite identificar factores de riesgo que son definidos como aquellas situaciones que directa o indirectamente pueden afectar el estado maternofetal. La detección temprana de dichos factores y su manejo adecuado pueden modificar o prevenir un pobre resultado perinatal ${ }^{15} \mathrm{y}$ un buen cuidado prenatal es una de las estrategias más importantes para disminuir la mortalidad perinatal ${ }^{3}$. La variable no diligenciada conlleva de por sí una situación riesgosa puesto que se está asumiendo el peligro potencial de la probabilidad de su presencia lo cual implica el riesgo de dejar de tomar ciertas medidas orientadas a corregirla.

Las instituciones deben exigir a sus médicos el completo y correcto llenado de la información tanto del control prenatal como de las remisiones, contraremisiones y de los eventos finales del emba- 
razo y deben además crear sistemas que operen en la recolección de la información de los resultados perinatales, pues es el único mecanismo que permite evaluar el impacto de sus programas sobre la morbimortalidad materna y fetal.

Siendo los embarazos de alto riesgo responsables de $70 \%$ a $80 \%$ de la morbilidad perinatal ${ }^{8,16}$, la historia prenatal debe constituirse además en un instrumento útil que permita seleccionar las pacientes de un bajo riesgo para ser atendidas en niveles menos complejos y remitir las pacientes con embarazos de alto riesgo a un nivel tecnológico capacitado para resolver situaciones de mayor complejidad. Aunque esta estrategia es lógica y busca la racionalización de los recursos, su eficiencia depende del buen diligenciamiento de la historia, de la acertada detección de situaciones de riesgo, de la capacitación del personal que realiza el control, de la adecuada dotación de las instituciones y de un buen funcionamiento de los sistemas de remisión.

La disminución de la mortalidad perinatal observada en el presente estudio y que confirma los resultados de investigaciones previas ${ }^{11-12}$, es el efecto de un mejor cuidado en la atención prenatal, una mayor identificación de los factores de riesgo y una mejor detección de la patología del embarazo, logrados al utilizar la historia clínica como un instrumento de evaluación de calidad.

Concluimos que el buen diligenciamiento de la historia clínica, la aplicación de un instrumento de control de calidad y la supervisión, conllevan a mejorar los resultados perinatales, además de que la acción de involucrar a los médicos y enfermeras en los programas de evaluación de la calidad de la atención es una estrategia que genera mayor compromiso y actitudes mas positivas del personal de salud en la atención de la mujer embarazada.

\section{AGRADECIMIENTOS}

Los autores desean hacer un especial reconocimiento a la Sociedad Colombiana de Obstetricia y Ginecología, a la Sociedad Vallecaucana de Obstetricia y Ginecología y sus anteriores presidentes doctor Jaime Ferro Camargo y María Cecilia Arturo por su interés y su aporte a este estudio. Igualmente desean agradecer la participación y colaboración de los doctores José Castro, Amparo Pungo, Otto Citely, Ángel Erazo, Lida Mera, Marcela Zuñiga, Sandra Agredo; enfermeras, Luz Marina Camacho, María Mercedes Ortega, María Eugenia Prado, funcionarios del Seguro Social; a los doctores Edgar Parra, Álvaro Tobón, Olinde Mejía, Jairo Cabezas, Vilma Constaín, Licen- ciada Martha R. Vargas, enfermeras Flor Fernández y Noralba Maca, funcionarios del Servicio de Salud; a los obstetras doctores Héctor Suárez, Guillermo Garrido, Eyder Burbano, Policarpo González y Francisco Valencia, miembros de la Sociedad Caucana de Obstetricia y Ginecología; al doctor Sofonías Yacup (q.e.p.d.) director del Hospital San José, doctores Francisco Otoya y Jorge Orozco, funcionarios de la misma Institución; al doctor Edgar Orejuela director del Hospital Susana López de Valencia y a todos aquellos que contribuyeron con su invaluable aporte a la culminación de la presente investigación.

\section{BIBLIOGRAFÍA}

1. Centro Latinoamericano de Perinatología. Incidencia de bajo peso al nacer y mortalidad perinatal en America Latina. Salud Perinatal 1983; 1: 3-6.

2. Moreno A. Mortalidad Perinatal y Materna. Revista Colombiana de Obstetricia y Ginecología 1990; 41(1): 33-38.

3. Dott AB.,Fort AT.The effect of maternal demographic factors on infant mortality rates: sumary of the findings of the Louisiana infant mortality study. Par I. Am J. Obst Gyn 1975; 123: 847.

4. World Health Organization (WHO) and United Nations Children's Fund. Revised 1990 estimates of maternal mortality: A new approach by WHO and UNICEF (Geneva) WHO. Apr. 1996.

5. Vélez JE, Dávila L. Mortalidad materna institucional en Caldas 1990 - 1994. Revista Colombiana de Obstetricia y Ginecología 1996; 47(1): 23-27.

6. Sarmiento M. Factores de riesgo obstétrico en pacientes recibidas para atención del parto en dos instituciones de primer nivel en Santafé de Bogotá, Colombia, 1993. Revista Colombiana de Obstetricia y Ginecología 1997; 48(4): 249-254.

7. Gómez P.,Ruiz N.,Pulido J. Mortalidad materna en el Instituto Materno Infantil de Santafé de Bogotá D.C. 1985 -1989. Revista Colombiana de Obstetricia y Ginecología 1993; 44 (1): 39-47.

8. Huaman M. Influencia de los programas de planificación familiar en la mortalidad perinatal. Revista Colombiana de Obstetricia y Ginecología 1997; 48 (2): 135-137.

9. Rojas O. Colombia: Perfil de salud reproductiva 1990. Revista Colombiana de Obstetricia y Ginecología 1991; 42(4): 279 -284.

10. Uriza G, Barragán E. Mortalidad Perinatal. Revista Colombiana de Obstetricia y Ginecología 1986; 37(2): 91.

11. Pazmiño S. Mejoramiento continuo del programa de atención prenatal en Instituciones del Seguro Social y de la Secretaría de Salud de Cali. Revista Colombiana de Obstetricia y Ginecología 1997; 48(1): 39-46.

12. Pazmiño S, Amastha F, Perdomo C y col. La calidad del control prenatal como garantía de salud de madres y recién nacidos. RevistaColombianadeObstetriciayGinecología1998;49(4):190195.

13. Ministerio de Salud. Decreto $N^{\circ} 2174$ de noviembre 28 de 1996. Firmado María Teresa Forero de Saade. Ministra de Salud.

14. Schwarcz R. y col. Historia Clínica Perinatal simplificada. Propuesta de un modelo para la atención primaria de baja complejidad.OPS/OMS/CLAP. Publicación científica 973, 1983.

15. Quintero C. Identificación y control del embarazo normal y de alto riesgo. En: R. Cifuentes, ed. Obstetricia de alto riesgo, cuarta edición, Cali: Aspromédica XYZ ImpresoresLtda1994: 83-93.

16. Hobel CJ., Hyvarinen MA, Okadda DM., et al. Prenatal and intrapartum high-risk secreening. Am J Obst Gyn 1973; 117: 1. 